Voix et Images

volxetimages

\title{
Blanche forcée ou la problématique du voyage chez Beaulieu
}

\section{Agnès Whitfield}

Volume 5, numéro 1, automne 1979

Jacques Godbout

URI : https://id.erudit.org/iderudit/200194ar

DOI : https://doi.org/10.7202/200194ar

Aller au sommaire du numéro

\section{Éditeur(s)}

Les Presses de l'Université du Québec

ISSN

0318-9201 (imprimé)

1705-933X (numérique)

Découvrir la revue

Citer cet article

Whitfield, A. (1979). Blanche forcée ou la problématique du voyage chez Beaulieu. Voix et Images, 5(1), 165-176. https://doi.org/10.7202/200194ar d'utilisation que vous pouvez consulter en ligne.

https://apropos.erudit.org/fr/usagers/politique-dutilisation/ 


\section{Blanche forcée ou la problématique du voyage chez Beaulieu}

Depuis le titre même de la série d'ouvrages que Beaulieu prévoit intituler les Voyageries et dont Blanche forcée ${ }^{1}$ ne constitue que le premier volume, aux multiples allusions textuelles, le lecteur ne peut se méprendre sur le sens de l'histoire de Job J Jobin. Il s'agit bien, comme le déclare le héros lui-même suivant l'humeur et le moment, d'un « voyage" (BF 13), ou encore d'une "voyagerie libidineuse" (BF 98), voire d'une "odyssée" (BF 63). Voyage qui amène Job et son amie Blanche de la rue Saint-Denis à Montréal à un petit "shaque qui fait face au Rocher de Percez [sic]" (BF 19).

La destination de Job et de Blanche n'a rien d'arbitraire. Lieu de la première rencontre du couple, la Gaspésie est aussi le foyer de toute la tribu des Jobin. Le grand-père de Job que le héros affectionne en particulier, était un vieux loup de mer « qui connaissait le St. Laurent [sic] comme sa poche pour avoir bourlingué dans le golfe et bien au-delà " (BF 27). Paysage réel à conquérir que le héros prend du plaisir à nommer et à explorer, la Gaspésie représente également tout un héritage affectif et culturel à déchiffrer et à assumer. Ainsi Job va-t-il parcourir le pays de Matapédia à l'Anse-au-Griffon, tout comme il essaie de connaître plus profondément Blanche, pour mieux en prendre possession. En même temps, fidèle à sa «profession d'océanologue» (BF 13) (il prépare un documentaire sur les baleines) Job renoue connaissance avec son grand-père, et par-dela lui, avec les «vieux pêcheurs de baleines" (BF 37 ) et la magnifique "Ventre-de-soufre".

Le sens du voyage de Job rejoint ainsi plusieurs niveaux d'analyse suivant que l'on étudie sa portée nationaliste, mythique ou affective. Pour sa part, Job hésite à trop anticiper sur l'issue de son Odyssée: "Le voyage fait que commencer ", constate-t-il au début du livre, " et mon désir de Blanche et de moi-méme, au centre duquel il y a le sens de l'entrelacs que forment mes initiales, mais peut-être davantage, comment savoir du fin fond de mon incompétence?" (BF 13) L'essentiel pour le héros semble se résumer dans une formule laconique: "Je voudrais pas qu'il y ait de malentendu", déclare$t$-il, « je cherche seulement à venir dans le monde du sens " (BF 15). Comment interpréter cette quête du sens ? Quelle est la signification de la «voyagerie" gaspésienne de Job? 
Or, loin d'étre particulier à Job, le voyage, au sens large du terme, constitue un élément important de la vie affective de plusieurs héros de Beaulieu. Ainsi, avant d'analyser la démarche de Job, importe-t-il d'étudier brièvement celle des héros beaulieusiens précédents. Chez ces derniers le voyage se présente le plus souvent sous la forme de la fuite. N'en pouvant plus de Montréal qu'il qualifie de "tour de Babel "2, Satan roule son tonneau «aussi loin que possible» (BF 20) et s'installe seul comme Diogène, à côté " de la petite source intarissable du rocher" (M 42). Dans Race de Monde!, le narrateur, Abel Beauchemin, quitte la maison familiale pour vivre dans une petite pièce rue Saint-Denis à Montréal. Sa fuite est donc symbolique plutôt que réelle et implique la famille davantage que la ville. Mais dans le cas de Jos, frère aîné d'Abel et même de Steven, leur frère poète, le voyage prend de l'ampleur. Jos part un beau jour sans rien dire (Nous apprenons dans Jos Connaissant ${ }^{3}$ qu'il traverse le Canada, vivant, destination inconnue, à la bonne étoile (JC 40)). Quant à Steven, par une correspondance curieuse qui associe la littérature à l'évasion, il quitte le Québec à la fin de Race de Monde! pour recevoir un prix littéraire à Paris où il reste d'ailleurs pendant plusieurs années.

Dans le cas de Malcomm Hudd, Montréal est la destination plutôt que le point de départ. Malcomm fuit sa maison de campagne pour échapper à la colère de sa femme. Une fois en ville, toutefois, il rêve d'aller à New-York en compagnie d'une nouvelle amie, Ricki. Ce deuxième départ prend l'allure d'un voyage de libération pour le protagoniste; mais, bien qu'il domine l'intrigue du roman, il demeure au stade du projet. Par contre, Momo, le héros d'Oh Miami Miami Miami ne vit pas que de rêves. Au moment où s'ouvre le roman, il s'en va faire peau neuve sur les plages floridiennes.

Toutefois, dans d'autres romans de Beaulieu, le voyage n'est pas synonyme de fuite ou de départ, mais implique plutót un retour. C'est le cas, par exemple, de Jos Beauchemin qui, revenu de sa fugue trans-canadienne dans Jos Connaissant, est amené par l'enterrement de sa mère, à quitter Montréal pour la terre de son enfance, ce « monde connu, apaisant, immobile de jadis à Saint-Jean-de-Dieu et à Trois-Pistoles" (JC 128). Cette nostalgie de l'enfance se poursuit par personne interposée dans les Grands-pères ${ }^{4}$ où Jos, s'il était présent à la narration, pourrait suivre son grand-père maternel, Milien, dans le pays d'enfance de sa mère. Milien retourne également à la maison où il avait lui-même vécu toute sa vie (GP 32), en plus de faire un autre voyage encore. En compagnie de trois amis, il part en voiture pour Montréal, dans l'espoir de ramener ses enfants perdus chez lui et de "reconstruire le clan menacé par l'éloignement" (GP 107). Dans le cas du héros d'Un rêve québécois, il y a aussi voyage de retour, mais au logis conjugal plutỏt qu'à la maison d'enfance.

Quel que soit le but du voyage envisagé ou entrepris par le héros beaulieusien, il est voué, on dirait presque a priori, à l'échec. Le dénouement du roman beaulieusien n'est jamais heureux. Satan Belhumeur est tué par les Petits-Gibettiens. Malcomm Hudd se suicide. Lémy, Momo et même Jos, 
comme nous l'apprenons dans Don Quichotte de la démanche sombrent dans la folie. Milien éprouve une angoisse qui frôle aussi la folie avant sa mort. Seul Abel Beauchemin connaît un sort moins radical.

Malgré leur diversité apparente, les différents motifs susceptibles de provoquer chez le héros beaulieusien le désir de partir, présentent plusieurs éléments constants. De façon schématique, on pourrait dire que lorsque le voyage se pose comme départ ou fuite, il provient du désir du protagoniste de se libérer de certaines contraintes, qu'elles soient d'ordre familial, social ou sexuel. Par contre, lorsque le voyage prend la forme d'un retour, il se présente comme la réintégration au foyer, ou, au sens plus large, comme une quête des racines.

Or, de prime abord, l'aventure gaspésienne de Job semble appartenir à cette dernière catégorie de voyages, en ce sens qu'elle se définit non pas comme fuite, mais comme retour. Seulement, les mobiles du retour ne sont plus les mêmes. Job vise en fait deux objectifs précis. D'une part, il sent que son bonheur avec Blanche est menacé et il veut remédier à la situation en revenant aux lieux de leur première rencontre. Il y a donc ce que nous appellerons une quête de l'amour. D'autre part, il a besoin de revoir la Gaspésie pour terminer ses recherches sur les baleines. Si celles-ci aboutissent à une sorte de quête des racines, en amenant Job à se pencher sur son passé et sur celui de sa famille, elles ne se définissent pas comme telles.

Blanche forcée marque-t-il donc un tournant dans le voyage beaulieusien? Nous allons voir que les deux objectifs de Job recèlent, en fait, la même problématique de fuite et de retour que nous avons découverte chez les autres héros de Beaulieu. Qui plus est, l'analyse de Blanche forcée va nous permettre de mieux comprendre les fondements de cette problématique.

Au niveau structural, l'aventure amoureuse de Job occupe une place privilégiée. C'est elle qui détermine la durée de la narration et, en grande partie, le déroulement de l'histoire ou de la diégèse ${ }^{5}$. Job commence à raconter son histoire le lendemain de son retour avec Blanche en Gaspésie (BF 22-23) et il la termine à la fin de la même journée en décrivant le suicide de sa maîtresse. En nous fondant sur les déplacements du couple pendant cette journée, nous pouvons diviser la durée narrative en deux "moments" presque identiques. Le soir, comme l'après-midi, Blanche et Job font une promenade en bateau, prennent un verre au bar et rentrent au "shaque" où Blanche ne manque pas à chaque fois de s'enfermer dans la salle de bains. Sur le plan affectif, cependant, il s'agit de deux phases distinctes qu'il importe d'examiner de plus près.

Dans la phase initiale, Blanche et Job tentent de se réconcilier. Seulement, ils n'arrivent pas à « se reprendre en main [...] dans le premier lieu du rite" (BF 20), malgré sa puissance évocatrice. Le passé immédiat prend le pas sur le passé plus éloigné. Job ne peut s'empêcher de repenser à " cette nuit d'épouvantement " qu'il a vécue avec Blanche «dans la chambre mauve en Mattavinie» (BF 29). C'est cette crise de Blanche qui semble constituer 
l'origine des difficultés du couple. "Ça nous a même forcés à fuir la vieille maison de Mattavinie" déclare Job, “Mais Gaspeg, est-ce mieux? " (BF 31).

L'ambivalence croissante que Job éprouve à l'égard du retour en Gaspésie, du moins pour ce qui est de ses rapports amoureux, a des répercussions intéressantes sur le plan spatial. La rentrée au « shaque" est remise à plusieurs reprises (BF 23 et 29). Job établit une correspondance entre la maison de Mattavinie et le shaque et craint de provoquer, semble-t-il, en réintégrant celui-ci, une répétition de la crise de Blanche. S'il arrive, bien qu'à contre-cceur, a franchïr le seuil du logis (BF 33), son angoisse n'en diminue pas pour autant. A ses yeux, le "shaque», tout comme la maison de Mattavinie devient l'expression spatiale de sa claustration amoureuse:

C'est comme si on était suspendus quelque part dans l'espace [constate Job] qu'on flottait dans du liquide, sans pesanteur, incapables de faire ces gestes qui mettraient fin au malentendu. Quand retrouverai-je Blanche et autrement que dans cette petrification où elle me fait l'effet d'être, à des milliers d'années-lumière de moi, débris du monde, noyau éclaté de notre affection circulaire (BF 60).

Lorsque Blanche vit de nouveau a exactement comme il y a trois jours dans la maison de Mattavinie, à peu près la même scène" (BF 65), Job, exaspéré, ne pense plus qu'à fuir le "shaque... déjà contaminé et déjà hostile" (BF 65).

La première phase du roman ramène donc les deux partenaires, malgré leur déplacement physique, au point de départ, à savoir la crise de Blanche en Mattavinie. Mais cette phase permet déjà de mieux cerner le sens du retour en Gaspésie. Il s'agit en fait d'un voyage qui est à la fois retour et fuite: retour au lieu de la première rencontre, mais fuite de la maison de Mattavinie avec tout ce qu'elle implique, sur le plan affectif, de troublant et de pénible.

Dans la seconde phase du roman, la double signification du voyage gaspésien ainsi que le sens de ces deux lieux-clés du couple, se précisent. Le héros se penche alors sur les débuts de sa liaison avec Blanche. Mais, comme le déclare Job, «la vérité vraie, c'est que Blanche est pas la dans les débuts, recluse dans sa Joliette sans histoire, moi campeur solitaire sur cette petite butte fesant [sic] face au Rocher de Percez" (BF 67). Aussi, Job va-t-il raconter parallèlement, son propre voyage en Gaspésie et celui de Blanche jusqu'au moment où, ces deux voyages convergeant, il aborde le récit proprement dit de sa liaison. En plus d'une description de la première rencontre des amants, nous avons donc affaire à un autre voyage, à savoir le premier voyage du héros en Gaspésie.

Ce voyage solitaire rappelle de façon frappante, le voyage-fuite des autres protagonistes beaulieusiens. Comme Malcomm Hudd, Job part à la suite d'une dispute avec sa femme, France. Le voyage lui permet de se libérer des contraintes conjugales et de faire peau neuve:

Rien de ce que tu as vécu a vraiment été vécu [se dit Job], tu viens tout juste de naître, greyé d'une nouvelle paire d'yeux. Vois et nourris-toi du vieux monde bien au chaud dans la chaleur de Gaspeg (BF 79). 
Se complaisant dans sa nouvelle liberté, Job s'installe dans sa petite tente bleve, tout comme Satan dans son tonneau.

Chose curieuse, loin d'accroître le bonheur du héros, l'arrivée de Blanche en Gaspésie semble plutôt y porter atteinte. Notons que sur le plan de la remémoration, Job ne s'empresse pas de narrer sa première rencontre avec Blanche. Bien au contraire, il profite de tous les prétextes pour la remettre à plus tard. II décrit en détail chaque étape du voyage de Blanche et de son amie Mina, le long du Saint-Laurent; il divague, se laissant aller à des souvenirs de France et de sa fille, Una. Méme lorsque les deux filles, rendues enfin en Gaspésie, rejoignent le champ visuel du héros, la rencontre est différée. "Je cherchais mais Mina et Blanche sont difficiles à trouver" (BF 95), se dit le héros, ou encore, « je cherche toujours Blanche et Mina [...] Peut-être [qu'elles] ont pas embarqué après tout" (BF 96).

Toutefois, lorsqu'enfin, le héros se décide à narrer la première rencontre, son récit se révèle tout aussi lacunaire. Job constate à plusieurs reprises qu'il " ne sai[t] pas ce qui s'est passé" (BF 120) ou bien qu'il « ne saurai[t] pas dire comment ça s'est terminé cette nuit-lá dans la petite tente bleue" (BF 125). D'ailleurs, le rôle qui revient à Blanche sur le plan sexuel est accessoire. Job fait davantage état de ses rapports avec Mina. Dans le contexte de cette "non-rencontre», le retour en Gaspésie perd toute valeur réparatrice. De conclure le héros:

On est écartés de belle façon, sans rien à dire qui dissimulerait le malentendu et percerait le mystère de cette nuit d'il y a trois ans. Je sais plus à quoi remonter (BF 119).

De nouveau, l'évolution affective du héros s'accompagne, sur le plan spatial, par une détérioration de la situation des deux partenaires au moment de la narration. Assis sur la grève avec Blanche, au début de la seconde phase du roman, Job constate quelques pages plus loin, qu'ils « auraient " pu choisir un autre lieu pour parler de tout ça» (BF 77). Plus tard, "effrayés tous les deux par ce qui monte vers [eux] et [ne leur] laisse aucun répit" (BF 90), ils quittent la grève pour monter dans une petite barque. Alors, ils deviennent progressivement plus “ mal à l'aise" (BF 94) au point où ils pensent même rentrer au "shaque". Pourtant, Job se rend compte qu'un tel geste ne pourrait qu'aggraver son angoisse: "Il suffira qu'on revienne au shaque, qu'on s'allonge l'un à côté de l'autre, pour recréer la vieille maison de Mattavinie qui est comme un poids dans ma poitrine” (BF 99).

A mesure que le récit se déroule, le voyage, qui se pose d'abord comme retour, se définit donc de plus en plus comme fuite - fuite de Mattavinie, sortie précipitée du "shaque", remise de la remémoration de la première rencontre, refoulement final des rapports entre Blanche et Job lors de cette rencontre. Le héros ne fait que tourner en rond, obsédé de plus en plus par la maison de Mattavinie. En voulant sortir du labyrinthe, Job s'empêtre davantage. Tout le ramène inévitablement à cette crise d'angoisse en Mattavinie. Sur le plan spatial, le couple ne peut plus, après la fermeture du bar, remettre 
leur retour au "shaque". En même temps, la remémoration que Job poursuit de façon plus ou moins chronologique rejoint le séjour en Mattavinie.

Que se passe-t-il en Mattavinie qui fait tant basculer le sens du voyage gaspésien? Paradoxalement, tout comme le retour en Gaspésie, le premier voyage du couple en Mattavinie est aussi une sorte de fuite qui a pour but de faire oublier à Job et à Blanche "cette scène disgracieuse dans Joliette" (182):

On s'en allait au devant d'une aventure pas comme les autres, dont on avait besoin dans notre amour.

C'était un lieu qu'on cherchait, pour mieux habiter notre désir, à l'étroit tout autant au Tourist Room pres de l'Ontario que dans Joliette [...] Voilà pourquoi, ce jour-là, la Volkswagen roulait vers les montagnes de Mattavinie (179).

Cette fois-ci, pourtant, la fuite semble couronnée de succès. Pour la première fois, le couple a un vrai foyer que Job prend du plaisir à aménager, "pour que la vieille maison de Mattavinie [leur] appartienne vraiment » (182). Seulement, le nid que le héros construit, c'est surtout le sien propre - fait d'objets et de souvenirs aptes à reconstituer un passé essentiellement familial. Job passe souvent ses journées dans la "vieille barque radoubée du grand-père Job J" qu'il transforme “en une espèce de petit atelier" (187). En même temps, il retrouve le plaisir de a descendre le fleuve pieds nus comme dans [son] temps d'enfance" (87).

Quant à Blanche, elle ne peut partager la vie du héros en Mattavanie que si elle accepte de jouer le jeu, de lui faciliter ce retour à l'enfance, voire à la matrice:

Ou encore [dit Job] je pars, monté sur mon tracteur comme un Quichotte. C'est ce qui est derrière la petite montagne d'arbres qui m'attire, ce maigre ruisseau et ce petit pont où coule mon fleuve de Mattavinie. Je ne reviendrai qu'avec la tombée du soir, exubérant, demandant à Blanche pourquoi elle refuse d'aller avec moi. Je dis: «la terre est si chaude Blanche, comme une peau de femme, pleine d'odeurs de femme, j'aimerais ça y dormir avec toi sur moi. "Elle répond: "demain" [...] Mais c'est pas arrivé une seule fois [...] Je comprenais pas, j'avais beau m'y prendre par les mains et par les pieds [...] couinant comme un rat: “ Blanche j'ai besoin de toi. Au centre des eaux tumultueuses, il y a cette île avec le petit lac au milieu et c'est ça que je veux atteindre» (41).

Tant que Job peut faire durer l'illusion de ce retour à la terre et à l'eau matricielles par le truchement des correspondances spatiales (le ruisseau et le fleuve de Mattavinie, le petit lac), il est heureux en Mattavinie. La réussite provisoire de la maison de Mattavinie en tant que lieu intime, tient donc à un malentendu. Pour le héros, il ne s'agit pas d'un foyer conjugal mais plutôt d'une sorte de maison maternelle. 
Ainsi n'est-il pas étonnant que la crise de Blanche qui provoque le départ de Mattavinie ait des connotations cedipiennes. Bien que Job ne décrive jamais, de façon intégrale, la crise de Blanche, les allusions qu'il y fait, où il la confond d'ailleurs souvent à des crises subséquentes, font toutes état des rapports sexuels que Blanche entretient, ou croit entretenir, avec son père ou une figure paternelle. Dans le passage suivant, nous voyons la constellation typique. Blanche, au bord d'une crise, décrit à Job les événements qui la tourmentent:

Elle était allée reconduire CatuLLe LasaLLe [l'amant de sa mère] à la porte mais il arrivait pas à sortir [...] « après je sais pas ce qui s'est passé Job J. Tout ce que j'ai su, c'est qu'il m'avait prise dans ses bras, me tenant serrée contre lui. Ses lèvres Job J. Ecrasant les miennes. Sa langue forçant mes dents. [...]

J'étais furieux [reprend le héros] tout s'était mis à tourner dans la chambre couleur caca d'oie de la rue St. Denis [sic]. J'étais debout, pris dans le filet que m'avait tendu Blanche, qui me laissait sans moyens, comme figé dans mon impuissance, arrivant seulement à balbutier: "après, après Blanche?" Elle a dit: "je sais pas, Job J. Je sais pas."

Tantót, sur la dernière marche de la véranda, c'est encore cette phrase qui est sortie de Blanche, comme une misérable lamentation déchirant le voile de Mattavinie pour ramener l'ignominie au centre du monde $(177-178)$.

La crise de Blanche n'est donc pas importante en elle-même mais plutôt par les sentiments qu'elle provoque chez le héros. En présence d'un rival d'aspect paternel, même si ce n'est, comme ici, qu'en pensée, Job ne peut plus vivre l'illusion de son retour au sein de la mère. Le " voile de Mattavinie" se déchire. Lorsque CatuLLe LasaLLe arrive en personne en Mattavinie, Job trouve la maison " comme dégonflée, rendue à sa réalité ", " une vieille maison qui allait rester là pour rien" (197).

D'autres indications confirment le caractère essentiellement œdipien des réactions de Job à l'égard des crises de Blanche. Par un concours de circonstances loin d'être fortuit, la crise de Blanche en Mattavinie survient précisément au moment où le grand-père paternel de Job, sur son lit de mort, conseille au héros de laisser tomber Blanche (165). La mort du grand-père, que Job considère cormme son vrai père par opposition au veule Jérémie J, n'enlève rien à cette interdiction. Au contraire, comme le héros le constate lui-même, «c'est cette mort qui a tout précipité. Avant on a été heureux Blanche et moi” (154).

Cette structure oedipienne sous-tend aussi les autres tentatives de fuite du couple. Avant la crise de Blanche en Mattavinie, il y a eu cette « scène disgracieuse" à Joliette, sans compter celle du “Tourist Room près de l'Ontario" (175) à laquelle le héros fait des allusions trop vagues pour que nous puissions en tirer des conclusions probantes. Nous avons donc affaire à trois fuites: celle de Joliette et celle de Mattavinie laquelle aboutit à la fuite, cette fois-ci sur le plan de la remémoration, de la première rencontre en 
Gaspésie. Chaque fois, le même problème se pose. Job attribue le départ à une crise de Blanche mais cette crise coïncide toujours avec l'arrivée d'un rival paternel qui empêche l'attachement matriciel du héros.

Dans le cas de Joliette, il y a surimpression de présences maternelles. Blanche vit avec sa mere et ses deux sœurs et Job trouve dans cette « tribu femelle" une " chaleur qu'[il] avai[t] jamais connue" (148). Son bonheur vient pourtant surtout de la présence de la mère de Blanche:

Peut-être ai-je été amoureux de la mére de Blanche [se dit Job] dans un bizarre transfert de tendresse. [...] C'étaient les odeurs de son corps qui me montaient à la tête dans lesquelles je reconnaissais Blanche, comme si on avait été dans l'avenir, comme s'il y avait eu un saut en quelque part, dans le temps, vingt ans passés comme l'éclair sans mémoire et qui fesaient qu'on était là la mère de Blanche et moi, dans ce soubassement sombre, assis à l'indienne, comme vieillis et sans souvenir de ce qui avait été, cherchant seulement à retenir le moment, à s'y glisser comme dans un sac (150).

Étant donné le caractère maternel de la maison de Joliette, il n'est pas étonnant que Job s'y trouve «à l'étroit" "surtout depuis que la mère de Blanche [a] pris cet amant mystérieux» (179). Celui-ci, déclare le héros, " avait viré la maison à l'envers et chassé l'apaisement que Blanche et moi on y trouvait» (179).

Y a-t-il un rapport entre l'échec des maisons de Mattavinie et de Joliette, en tant que lieu intime, et l'échec du retour en Gaspésie? Sans aucun doute, la conception que Job se fait du lieu de sa première rencontre avec Blanche présente plusieurs analogies avec les logis subséquents du couple. Assis dans la tente de Mina et Blanche, Job a l'impression qu'il se retrouve c comme dans un ventre, qu'il suff[it] de rester là, sans trop bouger, pour échapper à l'hostilité soudaine de Gespeg " (120). II raconte des histoires aux filles a pour qu'elles se sentent [...] tout a coup maternelles " (114). Dans cette ambiance matricielle, dans ce « ventre sacré " (120), Job découvre un « monde circulaire d'énergie", à l'abri des influences extérieures, qui rappelle de façon frappante, l'indifférenciation de l'état prénatal:

En fait, je crois pas avoir rien dit au sujet des corps cette nuit-là. Voilà ce que Blanche veut pas admettre.

Elle dit: « mais tu as parlé d'atomes, de leur indifférence. [...] Seulement c'était triste à entendre et que, par ce que tu disais tu avouais que tu étais un homme mort. Rien que de l'énergie qui se prend et se déprend pour oublier qu'il tourne à vide. [...] Tout est pareil, dans l'enjeu de l'espèce. On compte pas là-dedans disais-tu (121).

Dès le début même de sa liaison avec Job, Blanche est donc prise dans l'engrenage de la confusion, chez le héros, du sexuel et du maternel. L'absence de rapports sexuels suivis entre les deux partenaires qui nous a amenés à rejeter la première rencontre comme une "non-rencontre" trouve ainsi sa raison d'être. D'ailleurs, Job semble éprouver lui-même une certaine 
culpabilité qu'illustrent son refoulement des précisions corporelles et sa crainte de mourir dans "l'enjeu de l'espèce ". C'est cette structure cedipienne qui sous-tend l'ambivalence du héros à l'égard du lien de la premièré rencontre - ambivalence qui ne cesse de croitre à mesure que Job doit faire face, au Tourist Room, à Joliette et en Mattavinie, à des situations plus ouvertement triangulaires.

Examiné sous cet aspect, le retour en Gaspésie prend une signification nouvelle. Car le héros persiste à confondre sa recherche de l'intimité amoureuse avec une quête matricielle dont «l'île avec le petit lac au milieu» constitue, tout comme en Mattavinie, le symbole principal. "C'est pour cette raison ", constate le héros le soir au bar, " que Blanche et moi [...] on placote a côté de notre traque pour finir de vidanger tout ce qui constitue nos arrières et atteindre enfin cette petite portion de nous au beau mitan de laquelle se trouvent le lac et l'île inventée» (146). Mais le malheur pour Job, c'est que Blanche représente pour lui et le bonheur du retour au sein de la mère, et le cauchemar de l'interdiction œdipienne. Symbole d'une contradiction insoluble, elle lui sera toujours inaccessible:

Tous ces soirs si tant pareils depuis l'autre soir en Mattavinie et qui me ramènent à toi m'égarent [déclare Job à Blanche] Je saurai jamais où est le point [...] Les pièces tombent l'une après l'autre dans le désordre, dans ma fin de Blanche qui est mon amour cloué, surcloué et transgressé.

Les si beaux seins de Blanche, salures de ma tendresse et que je prendrais encore tant de fois dans mes mains, que je mordrai et têterai, entre lesquels je mettrais la vieille maison de Mattavinie, le rêve aladin se dévidant sur mon ventre plat et le lac calme inventé (94).

C'est parce que Job tient tant à ce rêve de retour à la matrice, que le voyage gaspésien est voué à l'échec. La « voyagerie libidineuse» est interdite au héros; l'Odyssée ne peut le ramener au pays natal. L'île dont il rêve, tout comme le bonheur de la première rencontre, n'est qu'un mirage qui se dérobe à mesure que Job s'en approche. Tiraillé par son attachement filial à Blanche, le héros doit errer entre la Mattavinie et la Gaspésie, entre son amour "transgressé" et le giron maternel, voué à un trajet circulaire, tantôt fuite, tantót retour.

Toutefois, en entreprenant son voyage en Gaspésie, Jọb avait un autre but qui ne concernait pas, directement du moins, ses rapports avec Blanche. Le héros tenait aussi à poursuivre ses recherches sur les baleines en vue de terminer son documentaire. Mais par un curieux transfert affectif, ces recherches vont lui permettre de sortir de son impasse amoureuse et spatiale, et de donner ainsi au voyage gaspésien un sens nouveau.

Bien que les baleines semblent avoir toujours fasciné le héros, il ne commence à s'y intéresser sérieusement qu'à partir du début de sa liaison avec Blanche. Cette coïncidence n'est pourtant que la première de toute une série de correspondances qui vont s'établir dans l'esprit de Job, entre sa 
maîtresse et les baleines en général, et la magnifique Ventre-de-soufre en particulier. Déjà, lorsque le héros rappelle l'installation de Blanche et de Mina dans la tente à cóté de la sienne face à Percé, il n'arrive pas à savoir si "l'espoir nouveau » qu'il ressent alors "vient de la petite tente bleue qu'[il] a vue avant même de sortir de la [s]ienne, ou de tout autre chose, du grand songe des baleines du golfe» (87).

Cette correspondance entre Blanche et les baleines provient d'une certaine équivalence que Job établit sur le plan affectif. "II y a Blanche", déclare-t-il, " il y a les baleines, il y a moi circonstancié et il y a tout ce qui se profile derrière et devant " (122). Chose intéressante, c'est la quête des eaux matricielles qui constitue le lien, pour Job, entre sa partenaire et les baleines. L'essentiel, pour lui, c'est a ce qui [I]'attire vers le fleuve et ce qui dort sous l'eau, Ventre-de-soufre épuisée par la longue marche des mers du Sud à l'île de l'Anticosti, et Blanche, et le corps de Blanche se laissant entrouvir sur la grève de Gespeg» (49).

Étant donné ce contexte affectif, il n'est pas étonnant que les baleines contribuent beaucoup, tout comme Blanche en sa qualité de présence maternelle, à l'intimité de la maison de Mattavinie. Job y retrouve une atmosphère "curieusement marine» (187). II appelle la maison Le Vautour d'après le vaisseau du vieux " corsaire Pierre Duval ", maitre de l'fle Bonaventure où Ventre-de-soufre a trouvé la mort. De même, les aménagements que Job effectue à l'intérieur de la maison ont pour but de mettre "enfin en ordre ossements de baleines, bouts de dents, harpons crochis, images céphalégiennes, chaînes de fer, vaisselle, agrès de goélette, étambots miniaturisés de baleinières" (40), bref, tous les objets qu'il a rassemblés pour son documentaire sur les baleines.

Or, contrairement à ce qui se passe dans le cas de Blanche, le grandpère de Job ne voit pas d'inconvénient à ce que son petit-fils s'intéresse aux baleines. Il l'encourage même. "N'est-ce pas lui", constate le héros, "qui m'avait mis sur la piste de Ventre-de-soufre?" (154). Ainsi, en l'absence de l'interdiction paternelle, les baleines se présentent-elles comme une sorte de solution de rechange lorsque Job se rend compte de l'impossibilité de son amour pour Blanche. II suffit d'écarter sa maîtresse pour retrouver l'intimité matricielle de Mattavinie:

J'avais transformé la vieille barque du grand-père Job en une espèce de petit atelier [rappelle le héros]. Amarrée au petit pont du ruisseau, avec la tente bleue pour m'abriter de la mouille, la dent noircie de Ventre-desoufre plantée devant, curieux totem du golfe que je retrouvais dans toutes ses odeurs. Sans Blanche, j'aurais été le plus content des hommes, à cause de cette immobilité dans laquelle je me laissais descendre et qui m'enveloppait presque avec tendresse, comme un mot femelle, ou bien comme un ventre nourricier, c'était mou et vaste, à se sucer les pouces tellement tout me devenait irréel, avec le paysage pour me forcer dans la modestie du corps. L'impression heureuse d'étre plus rien qu'un filet de vie, qu'un souffle harmonieux (187-188). 
Comme nous le voyons dans ce passage, c'est la dent de Ventre-desoufre, ce "curieux totem du golfe" qui, remplaçant en ceci Blanche, assure le bonheur du retour au sein de la mère. Le héros abandonne ainsi c l'image inusable de Blanche» (171), qui ne peut plus évoquer pour lui la joie de l'intimité matricielle, en faveur d'une "image sûre" (59), autrement plus efficace. A la lumière de ce transfert affectif, le suicide de Blanche qui marque la fin du roman n'a rien d'étonnant ni d'accessoire. II ne reste à Job qu'à "envaler [sic] par le travers [sa] mise à mort de Blanche" (197) pour pouvoir se vouer à cœur joie à une autre Blanche plus pure:

Sur la grève deux vieux marins d'eau douce ouvrent les bourroles pour écorcher vif l'anguille [déclare Job au dernier chapitre du livre] Cinquante pieds de mer se disent-ils lorsque Job J Jobin passe près d'éux courant après son souffle de Blanche (213).

Job sort ainsi de l'impasse dans laquelle il se trouve au bout de son aventure gaspésienne, par ce que nous pourrions appeler un saut dans l'imaginaire. Sans doute est-il bon que le héros retrouve une nouvelle Blanche dans l'univers imaginaire, car c'est là où il a le plus de chances de découvrir, à long terme, au moyen de la sublimation, une échappatoire. II n'empêche qu'il n'accomplit son tour de force qu'en renonçant à sa liaison avec la vraie Blanche. En ce sens, la partie n'est pas gagnée; elle est tout simplement remise. Job ne résoud pas la structure œdipienne qui sous-tend l'échec de ses rapports avec sa maîtresse ainsi que l'échec du voyage en Gaspésie. Seulement, même s'il n'arrive ni à fuir la maison de Mattavinie ni à regagner sa terre natale, tout n'est pas perdu. Grâce aux baleines, il est libre de poursuivre l'Odyssée.

Aussi, le voyage gaspésien se caractérise-t-il non pas, comme on pourrait le croire de prime abord, par deux quêtes, étant à la fois recherche de l'amour et des racines, mais par une seule, celle de la matrice. On ne peut certes, généraliser à partir d'un seul roman. Mais le rapport que nous avons découvert dans Blanche forcée entre les deux mouvements de fuite et de retour ouvrent néanmoins un nombre de voies intéressantes qu'il sera utile de poursuivre en ce qui concerne le thème du voyage dans d'autres romans de Beaulieu. $\mathrm{De}$ telles recherches s'imposent d'autant plus que la structure œdipienne sous-jacente au voyage gaspésien se retrouve chez d'autres héros beaulieusiens ${ }^{6}$. Par ailleurs, le déplacement de la quête matricielle au profit de la thématique des baleines pourrait signaler un virement important dans l'orientation de la symbolique spatiale beaulieusienne qu'il importe d'élucider. Peut-être faudrait-il y voir le concours de circonstances, tant littéraires qu'affectives, à l'origine du culte que vouent les successeurs beaulieusiens de Job au Moby Dick de Melville. 
1. Beaulieu, V.-L., Blanche forcée, VLB éditeur, Montréal 1976. Désormais désigné par «BF".

2. Beaulieu, V.-L., Mémoires d'outre-tonneau, Éditions Estérel, Montréal 1968, p. 23. Désormais : " $M$ ».

3. Beaulieu, V.-L., Jos Connaissant, Éditions du Jour, Montréal 1970. Désormais: " JC".

4. Beaulieu, V.-L., les Grands-pères, Editions du Jour, Montréal 1971. Désormais : "GP".

5. Nous reprenons les mots « histoire "t a narration " tels que les emploie Gérard Genette qui désigne par "histoire" "le signifié ou le contenu narratif et par "narration" "l'acte narratif producteur et, par extension, l'ensemble de la situation réelle ou fictive dans laquelle il prend place" (Figures III, Seuil, Paris 1972, p. 72.).

6. Voir les travaux de Gérard Bessette (Trois romanciers québécois, Éditions du Jour, Montréal 1973 et "Oh Miami Miami Miami et la thématique beaulieusienne", Voix et Images du Pays IX (1975), p. 181-200) et l'article d'André Vanasse ( Victor-Lévy Beaulieu : A la recherche du mystère au bout de la Queue de Christ", Livres ot Auteurs québécois, 1972, p. 385-396). 